\title{
Efecto del oxígeno disuelto en la adsorción de oro en carbón activado ${ }^{(\cdot)}$
}

\author{
P. Navarro* e I. Wilkomirsky**
}

\begin{abstract}
Resumen Se estudió el efecto del oxígeno en la adsorción de oro en un carbón de uso típico en los procesos de carbón en pulpa (CIP) y carbón en lixiviación (CIL). El trabajo experimental se orientó al estudio del efecto del oxígeno en la forma y cantidad de oro adsorbido bajo diferentes condiciones de fuerza iónica, $\mathrm{pH}$ y concentración de oro de la solución acuosa. Se encontró que el oxígeno disuelto en la solución acuosa aumenta la absorción de oro en el carbón activado, siendo este efecto mayor en soluciones de baja fuerza iónica y menor cuando se trata de soluciones de elevada fuerza iónica. El pH y la concentración inicial de oro en la solución acuosa no modifican lo anteriormente planteado.
\end{abstract}

Palabras claves Carbón activado. Adsorción. Oxígeno. Fuerza iónica.

\section{The effect of the oxygen dissolved in the adsorption of gold in activated carbon}

\begin{abstract}
The effect of the oxygen dissolved on the adsorption of gold in a activated carbon such as these used for carbon in pulp (CIP) and carbon in leach (CIL) processes were studied. The research was oriented to dilucidate the effect of the oxygen dissolved in the gold solution on the kinetics and distribution of the gold adsorbed in the carbon under differents conditions of ionic strength, $\mathrm{pH}$ and gold concentration. It was found that the level of the oxygen dissolved influences directly the amount of gold adsorbed on the activated carbon, being this effect more relevant for low ionic strength solutions. The $\mathrm{pH}$ and initial gold concentration has no effect on this behavior.
\end{abstract}

Keywords Activated carbon. Adsorption. Oxygen. Ionic strength.

\section{INTRODUCCION}

Entre los distintos métodos para recuperar oro de disoluciones cianuradas, destacan por su mayor empleo la cementación con polvo de zinc y la adsorción con carbón activado. El interés por el carbón activado en la metalurgia extractiva del oro data de 1880 , fecha en que se propone su empleo como adsorbente a partir de disoluciones del proceso de cloruración. En 1890 se introduce la cianuración como alternativa al tratamiento de minerales de oro, encontrándose que tanto el oro como la plata podían ser adsorbidos de disoluciones obtenidas de este proceso. Sin embargo, su uso industrial se vio restringido debido a la falta de un método eficiente para recuperar el oro cargado en el carbón que, además, permitiese, su reutilización sin tener que recurrir, de esta forma, a la calcinación.

La situación planteada anteriormente cambió a partir del año 1952, con el desarrollo de los procesos de adsorción y electroobtención, proponiéndose nuevamente el empleo del carbón activado para recuperar el oro contenido en disoluciones originadas por los diversos procesos de cianuración, entre los que destacan actualmente, el de lixiviación en pilas, carbón en lixiviación (CIL) y carbón en pulpa (CIP $)^{[1-3]}$.

El proceso de carbón en pulpa (CIP) posee importantes ventajas comparativas sobre el de cementación de oro con zinc, dentro de las cuales se pueden destacar:

- La facilidad para adsorber el complejo que posee el carbón activado no se ve afectada por la presencia de algunos elementos en disolución, como complejos de cobre y níquel, los cuales desfavorecen la precipitación de oro con zinc.

- Las partículas de carbón se agregan directamente a la pulpa de cianuración, lo que evita los procesos de filtración y clarificación que se requieren con la cementación.

(•) Trabajo recibido el día 22 de enero de 1999 y aceptado en su forma final el día 10 de agosto de 1999.

(*) Departamento de Ingeniería Metalúrgica, Facultad de Ingeniería, Universidad de Santiago de Chile, Avenida Libertador Bernardo O’Higgins 3363, Casilla 10233, Santiago, Chile.

(**) Departamento de Ingeniería Metalúrgica, Facultad de Ingeniería, Universidad de Concepción, Casilla 53-C, Concepción, Chile. 
Las pérdidas de oro son significativamente menores que en una planta tradicional de cementación, por lo que el proceso CIP ofrece ventajas económicas, tanto por lograr mayores recuperaciones como en términos comparativos de costos de capital y operación.

Carbón activado es un término genérico que designa a una familia de materiales carbonáceos altamente porosos, que no pueden ser caracterizados por una fórmula estructural o por análisis químico, y que se caracteriza por ser un material que tiene una gran superficie específica, propiedad que le confiere una alta capacidad para retener en su superficie compuestos contenidos en disoluciones acuosas. En la actualidad, el carbón activado puede ser fabricado a partir de alguna materia prima carbonácea, como son diversos tipos de carbón (turba, lignito, carbón bituminoso, antracita), cáscaras de coco, huesos de durazno, madera, serrín, cáscaras de arroz, etc. ${ }^{[4-7]}$.

Con el desarrollo de procesos tales como carbón en pulpa (CIP) y carbón en lixiviación (CIL), se hizo necesario la fabricación de un material resistente a la abrasión para minimizar la producción de partículas finas de carbón causantes de pérdidas de oro y que, además, posean un tamaño adecuado que permita la separación de pulpa-carbón, sin que disminuya la cinética de adsorción de oro.

El mecanismo por el cual el carbón activado extrae el complejo $\mathrm{Au}(\mathrm{CN})_{2}^{-}$desde disoluciones de cianuración ha sido estudiado por diferentes investigadores, existiendo una diversidad de mecanismos propuestos que pueden explicar el fenómeno. Esta situación puede ser atribuida a los siguientes factores:

- Experimentos realizados con una variedad de carbones activados, es decir, fabricados a partir de diferentes materias primas.

- Carbones activados preparados bajo diferentes condiciones de activación.

- Estudios de adsorción-desorción de oro bajo diferentes condiciones experimentales y diferentes características de la disolución de cianuración.

A pesar de lo anteriormente expuesto, los mecanismos que explican la adsorción de oro en carbón activado, pueden ser agrupados en las siguientes dos grandes líneas ${ }^{[8-12]}$ :

- Adsorción sin cambio químico, es decir, como par iónico del tipo $\mathrm{M}^{\mathrm{n}+}\left[\mathrm{Au}(\mathrm{CN})_{2}^{-}\right]_{\mathrm{m}}$, donde $\mathrm{M}^{\mathrm{n}+}$ puede ser $\mathrm{Ca}^{2+}, \mathrm{K}^{+}, \mathrm{Na}^{+}, \mathrm{Li}^{+}, \mathrm{H}^{+}$, etc., y/o a través de intercambio de iones.

- Adsorción de $\mathrm{Au}(\mathrm{CN})_{2}^{-}$, con descomposición a otras especies del tipo AuCN.

De los resultados obtenidos, parece probable que todos los mecanismos de adsorción de oro en carbón activado son factibles de ocurrir en alguna medida, solo que alguno de ellos puede ser preponderante dependiendo de las condiciones experimentales utilizadas. De acuerdo con esto es importante conocer el efecto del oxígeno en la adsorción de oro.

En este trabajo se presentan los resultados obtenidos como consecuencia del efecto del oxígeno en la adsorción de oro, bajo diferentes condiciones experimentales de fuerza iónica y $\mathrm{pH}$ de la disolución acuosa, empleando carbón activado comercial que ha sido fabricado a partir de cáscaras de coco.

\section{PARTE EXPERIMENTAL}

\subsection{Materiales}

\subsubsection{Carbón Activado}

El carbón activado comercial que se utilizó es de forma cilíndrica y se fabrica a partir de cáscaras de coco. En la tabla I, se presentan sus principales propiedades físicas.

En la tabla II se muestra la distribución de poros en el interior del carbón activado. La superficie específica y la porosidad del carbón activado fue determinada por el método BET con nitrógeno.

De acuerdo con lo observado en la tabla II, el carbón utilizado en este estudio posee 3 a $5 \%$ de mesoporos (poros de transición) y entre 95 y $97 \%$ de microporos.

Tabla I. Propiedades físicas del carbón activado comercial empleado

Table I. Physical properties of comercial activated carbon used

\begin{tabular}{lc}
\hline \multicolumn{1}{c}{ Propiedad } & Valor \\
\hline Largo del cilindro, $(\mathrm{mm})$ & $5-7$ \\
Diámetro del cilindro, $(\mathrm{mm})$ & 3 \\
Superficie específica, $\left(\mathrm{m}^{2} / \mathrm{g}\right)$ & $900-950$ \\
Volumen total de poros, $\left(\mathrm{cm}^{3} / 9\right)$ & $0,43-0,47$ \\
Densidad real, $\left(\mathrm{g} / \mathrm{cm}^{3}\right)$ & 1,104 \\
Mesoporos $(8 \AA<\#<1000 \AA),(\%)$ & $3-5$ \\
Microporos $(¥<8 \AA),(\%)$ & $85-97$ \\
\hline
\end{tabular}

Rev. Metal. Madrid 35 (1999) 
Tabla II. Distribución de poros en el carbón activado

Table II. Pore distribution in the activated carbon

\begin{tabular}{cc}
\hline Tamaño de los poros $(\AA)$ & Porcentajes de poros (\%) \\
\hline $1.000-100$ & $0,00-0,68$ \\
$100-50$ & $0,01-0,81$ \\
$50-20$ & $1,11-1,79$ \\
$20-15$ & $1,24-1,70$ \\
$15-10$ & $2,80-5,82$ \\
$10-9$ & $2,39-4,08$ \\
$9-8$ & $3,30-5,75$ \\
$8-7$ & $5,96-7,20$ \\
$7-6$ & $6,81-8,34$ \\
$6-5$ & $9,83-14,17$ \\
$5-0$ & $53,31-63,71$ \\
\hline
\end{tabular}

\subsubsection{Disolución de oro}

Para la preparación de las disoluciones de oro se utilizó agua bidestilada y desionizada y reactivos químicos grado analítico, tales como: dicianoaurato de potasio $\left(\mathrm{KAu}(\mathrm{CN})_{2}\right)$, hidróxido de potasio $(\mathrm{KOH})$ y cloruro de potasio $(\mathrm{KCl})$. Se utilizaron disoluciones de 45, 55 y $105 \mathrm{mg} / \mathrm{l}$ de oro y de $6 \times 10^{-4}$ y $0,5 \mathrm{M}$ en potasio.

\subsection{Estudio experimental de adsorción}

Para todas las experiencias realizadas, el carbón activado se acondicionó previamente, a través de un lavado con agua bidestilada y desionizada durante 30 min, después se secó en una mufla durante 24 $\mathrm{h}$, a temperaturas que oscilaron entre 60 y $80^{\circ} \mathrm{C}$ y posteriormente fue mantenido en un desecador hasta su utilización.

En un reactor de vidrio, inmerso en un baño termostático de temperatura controlada, se introducían $250 \mathrm{ml}$ de disolución acuosa y 0,5 g de carbón activado, haciéndose burbujear oxígeno o nitrógeno en el seno de la solución, dependiendo del contenido de oxígeno disuelto requerido para cada experiencia. De la disolución acuosa se sacaba una muestra para análisis químico. El carbón activado se lavaba con agua bidestilada y desionizada durante $15 \mathrm{~min}$, para luego ser secado en una mufla durante $24 \mathrm{~h}$ a $70^{\circ} \mathrm{C}$. Esta metodología experimental fue llevada a cabo para cada uno de los puntos experimentales obtenidos, en las diferentes curvas cinéticas. Las condiciones experimentales en que se realizaron todas las pruebas de adsorción fueron:

$\begin{array}{ll}\text { masa de carbón } & : 0,5 \mathrm{~g} \\ \text { volumen de disolución } & : 250 \mathrm{ml} \\ \text { temperatura } & : 25^{\circ} \mathrm{C} \\ \text { velocidad de agitación } & : 500 \mathrm{rpm} \\ \text { tiempo de adsorción } & : 168 \mathrm{~h}\end{array}$

Los análisis de oro en solución acuosa fueron realizados usando espectrofotometría de absorción atómica y la medición de oxígeno disuelto se hizo mediante un electrodo de ion específico.

\section{RESULTADOS Y DISCUSION}

\subsection{Efecto del oxígeno disuelto en disolucio- nes de baja fuerza iónica}

Se pudo establecer que para disoluciones saturadas en oxígeno $(8 \mathrm{mg} / \mathrm{l})$, se produjo una mayor adsorción de oro en el carbón con relación a disoluciones de bajo contenido de oxígeno disuelto $(0,8 \mathrm{mg} / \mathrm{l})$.

Este efecto se hace mas pronunciado al emplear bajo contenido de potasio, es decir, $6 \cdot 10^{-4} \mathrm{M}$. En la figura 1 se muestra el porcentaje de adsorción de oro en función del tiempo, para una concentración inicial de oro de $55 \mathrm{mg} / \mathrm{l}, \mathrm{pH} 11$ y una concentración de ión potasio de $6 \cdot 10^{-4} \mathrm{M}$, bajo condiciones de saturación de oxígeno $(8 \mathrm{mg} / \mathrm{l})$ y con una disolución desoxigenada $(0,8 \mathrm{mg} / \mathrm{l})$. De igual forma, en la figura 2 se muestra la evolución del $\mathrm{pH}$ para los distintos tiempos de adsorción. Al emplear disoluciones saturadas en oxígeno y de baja fuerza iónica, además de operar el mecanismo de adsorción en la forma de par iónico $\mathrm{KAu}(\mathrm{CN})_{2}$, actúa de manera paralela un mecanismo de tipo electroquímico, activándose otros sitios superficiales

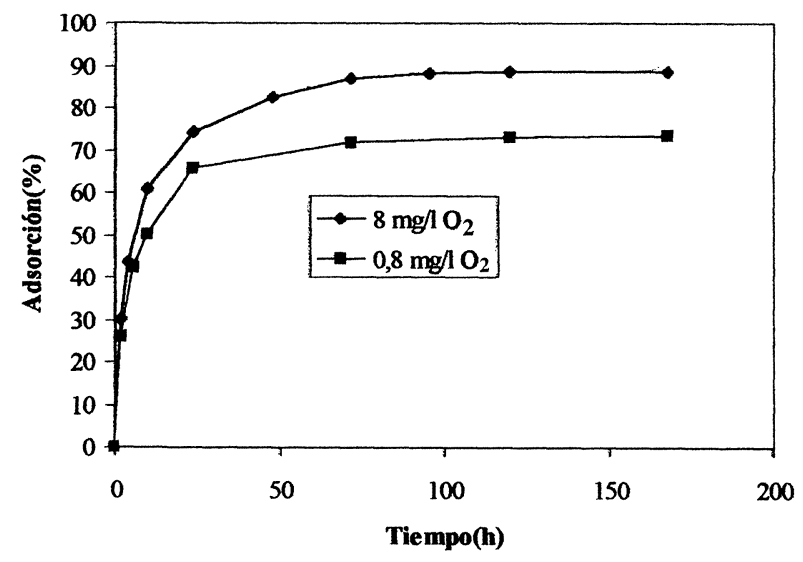

Figura 1. Efecto del oxígeno disuelto en la adsorción de oro en carbón activado para una concentración de $6 \cdot 10^{-4} \mathrm{M}$ de potasio.

Figure 1. Effect of dissolved oxygen in gold adsorption on activated carbon. Initial potassium concentration $6.10^{-4} \mathrm{M}$. 


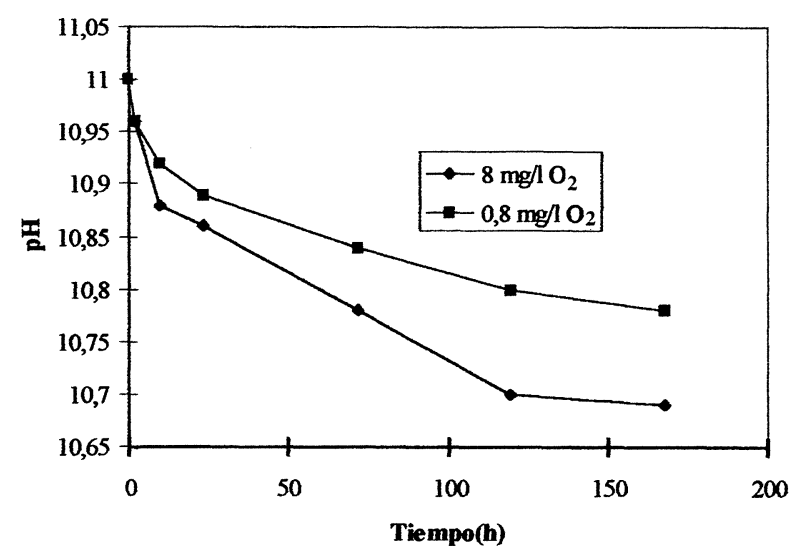

Figura 2. Evolución del pH en la adsorción de oro en carbón activado para una concentración de $6 \cdot 10^{-4} \mathrm{M}$ de potasio.

Figure2. Evolution of $\mathrm{pH}$ in gold adsorption on actived carbon. Initial potassium concentration $6 \cdot 10^{-4} \mathrm{M}$.

del carbón capaces de adsorber oro. De acuerdo con Mc Dougall y Hancock ${ }^{[13]}$ y Adams y Fle$\operatorname{ming}^{[14]}$, se produce la reducción del oxígeno en la superficie del carbón:

$$
\begin{array}{ll}
\mathrm{O}_{2}+4 \mathrm{e}_{\mathrm{e}}^{-}+2 \mathrm{H}_{2} \mathrm{O} \Leftrightarrow 4 \mathrm{OH}^{-} & \mathrm{E}^{\circ}=0,401 \mathrm{~V} \\
\mathrm{O}_{2}+2_{\mathrm{e}}^{-}+2 \mathrm{H}_{2} \mathrm{O} \Leftrightarrow 2 \mathrm{OH}^{-}+\mathrm{H}_{2} \mathrm{O}_{2} & \mathrm{E}^{\circ}=-0,146 \mathrm{~V}
\end{array}
$$

y la otra reacción de semicelda corresponde a la oxidación de la superficie del carbón, como por ejemplo, la oxidación del grupo cromeno:

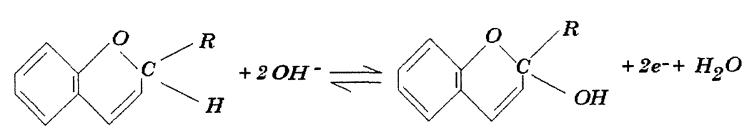

La estructura formada corresponde al ion carbonio, el cual puede actuar como un sitio de intercambio de iones:

$$
\overbrace{\mathrm{OH}^{-}}^{\mathrm{O}}+\mathrm{X}^{-} \rightleftharpoons{ }_{\mathrm{C}}^{\mathrm{R}}+\mathrm{OH}^{-}
$$

Para un tiempo de adsorción de 168 h de contacto entre el carbón activado y la disolución de oro, la razón K/Au en el carbón fue de 0,6 cuando se trabajó con $8 \mathrm{mg} / \mathrm{l}$ de oxígeno disuelto, lo cual está de acuerdo con los resultados obtenidos por Tsuchida et al. ${ }^{[15-16]} \mathrm{y}$ es consistente con el hecho de que bajo esas condiciones experimentales, es decir, en presencia de disoluciones saturadas en oxígeno y bajo contenido de ion potasio (baja fuerza iónica), ocurren los mecanismos de adsorción de par iónico y el de intercambio de iones.
Cuando se empleó 0,8 mg/l de oxígeno disuelto, la razón K/Au medida en el carbón después de $168 \mathrm{~h}$ de contacto fue siempre un valor cercano a 1 o levemente inferior, lo cual es consistente con el mecanismo de par iónico, como forma preponderante de adsorción de oro en el carbón. La disminución del $\mathrm{pH}$ en este caso puede ser atribuida a la adsorción de iones hidróxilos.

\subsection{Efecto de la concentración de oxígeno di- suelto en disoluciones de elevada fuerza iónica}

En la figura 3 se muestra el porcentaje de adsorción de oro en función del tiempo, para una concentración inicial de oro de $55 \mathrm{mg} / \mathrm{l}, \mathrm{pH} 11$ y una concentración de potasio de $0,5 \mathrm{M}$ y en la figura 4 se muestra volución del pH para los distintos

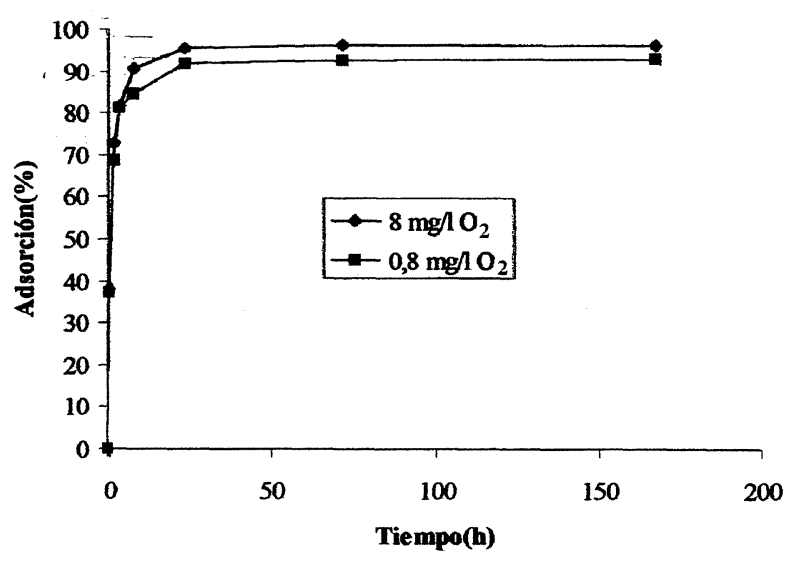

Figura 3. Efecto del oxígeno disuelto en la adsorción de oro en carbón activado para una concentración de 0,5 $\mathrm{M}$ de potasio.

Figure 3. Effect of dissolved oxygen in gold adsorption on activated carbon. Initial potassium concentration 0,5 M.

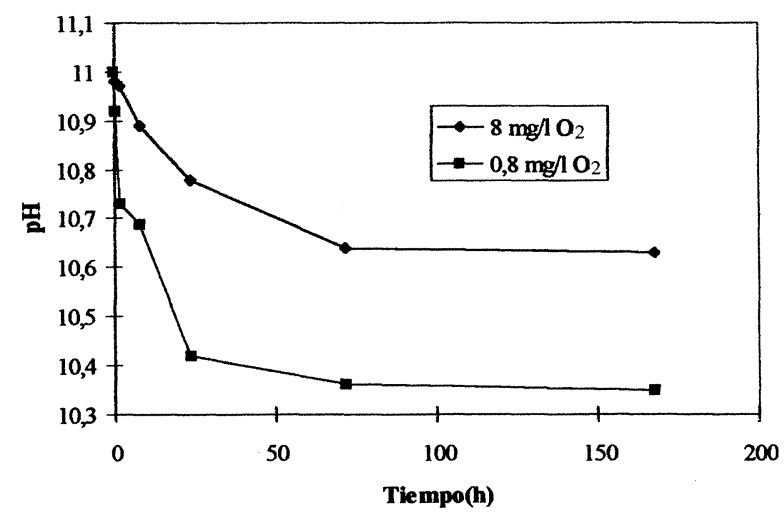

Figura 4. Evolución del pH en la adsorción de oro en carbón activado para una concentración de 0,5 M de potasio.

Figure 4. Evolution of $\mathrm{pH}$ in gold adsorption on actived carbon. Initial potassium concentration 0,5 M. 
tiempos de adsorción. En este caso, al emplear una disolución de fuerza iónica elevada, al término de $168 \mathrm{~h}$ de contacto, el efecto de experimentar con solución saturada en oxígeno $(8 \mathrm{mg} / \mathrm{l})$ se manifestó en solo un $4 \%$ de mayor adsorción de oro, en comparación con disoluciones de bajo contenido de oxígeno $(0,8 \mathrm{mg} / \mathrm{l})$. La variación del $\mathrm{pH}$ en este caso fue levemente mayor, con relación al que se trabajó con fuerza iónica baja.

Estos resultados indican que para disoluciones de elevada fuerza iónica, el efecto del oxígeno en la adsorción en el carbón activado se hace marginal, con lo cual, el mecanismo predominante es el de adsorción en la forma de par iónico como $\mathrm{KAu}(\mathrm{CN})_{2}$, debido a la elevada concentración de iones potasio en el medio acuoso. La formación de sitios de intercambio iónico, debido a la presencia de oxígeno disuelto, solo se manifiesta a través de un aumento del $4 \%$ en la adsorción de oro, después de $168 \mathrm{~h}$ de contacto.

Si se admite que las disoluciones de cianuración obtenidas en operaciones industriales de carbón en pulpa son consideradas como de elevada fuerza iónica, el oxígeno, además de aumentar el efecto de oxidación del cianuro libre, puede conducir a una condición más favorable en la adsorción de oro.

\subsection{Efecto del oxígeno a diferentes pH}

En la figura 5 se muestra la adsorción de oro en función del tiempo, para una concentración inicial de oro de $55 \mathrm{mg} / \mathrm{l}, 8 \mathrm{mg} / \mathrm{l}$ de oxígeno disuelto, dife-

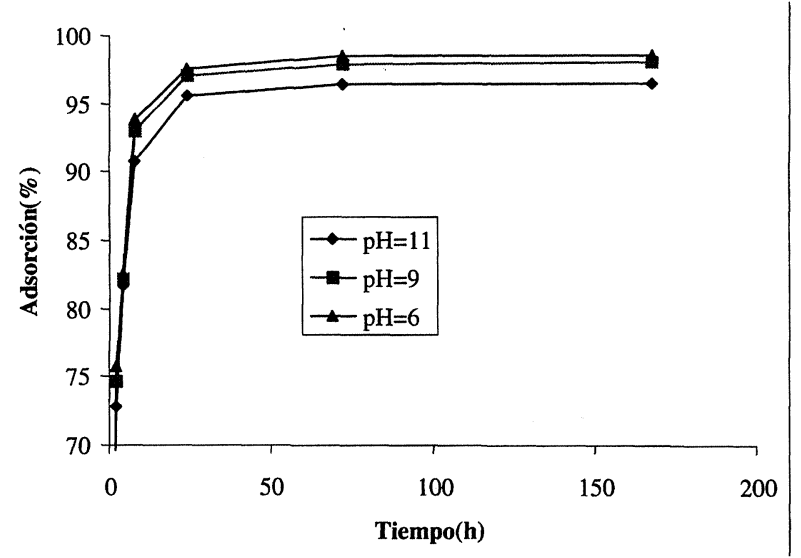

Figura 5. Cinética de adsorción de oro en carbón activado en disoluciones saturadas en oxígeno disuelto $(8 \mathrm{mg} / \mathrm{l})$, en función del $\mathrm{pH}$ de la disolución, y para una concentración inicial de potasio de 0,5 M.

Figure 5. Kinetics of gold adsorption on activated carbon from oxygen saturated ( $8 \mathrm{mg} / \mathrm{l}$ ) aqueous solutions of various $\mathrm{pH}$ and initial potassium concentration of $0,5 \mathrm{M}$.

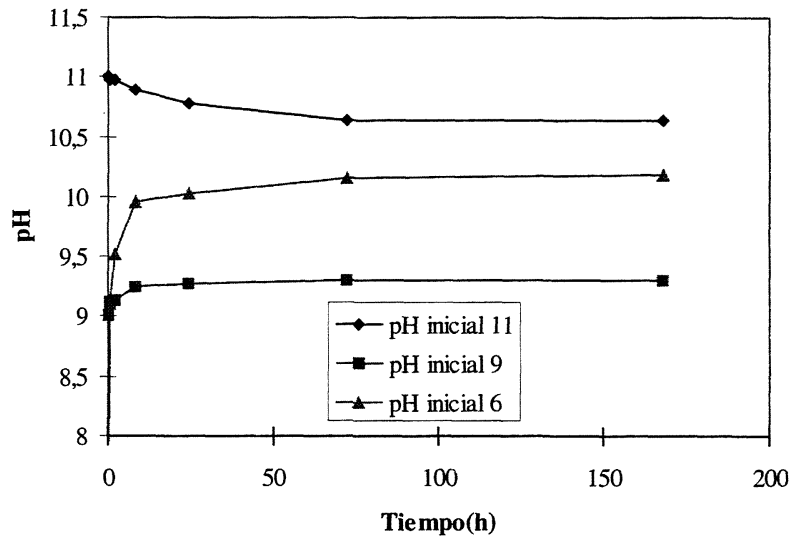

Figura 6. Evolución del pH en la adsorción de oro en carbón activado para una concentración de 0,5 M de potasio, 8 $\mathrm{mg} / \mathrm{l}$ de oxígeno disuelto $\mathrm{y}$ diferentes $\mathrm{pH}$ iniciales.

Figure 6. Evolution of $\mathrm{pH}$ in gold adsorption on actived carbon. Initial potassium concentration $0,5 \mathrm{M}$, dissolved oxigen $8 \mathrm{mg} / \mathrm{l}$ and diffrents initials $\mathrm{pH}$.

rentes $\mathrm{pH}$ de la disolución acuosa y una concentración de pótasio de $0,5 \mathrm{M}$ y en la figura 6 se visualiza gráficamente la evolución del $\mathrm{pH}$ para las diferentes condiciones iniciales. Se puede observar que al disminuir el $\mathrm{pH}$, aumenta levemente el porcentaje de adsorción de oro.

En la figura 7 se muestra la adsorción de oro en función del tiempo, para una concentración inicial de oro de $55 \mathrm{mg} / \mathrm{l}, 0,8 \mathrm{mg} / \mathrm{l}$ de oxígeno disuelto, diferentes $\mathrm{pH}$ de la disolución acuosa y una concentración del ion potasio de $0,5 \mathrm{M}$ y en la figura 8 se muestra la evolución del $\mathrm{pH}$ para las condiciones experimentales mencionadas. Al emplear disoluciones exentas de oxígeno disuelto, también se

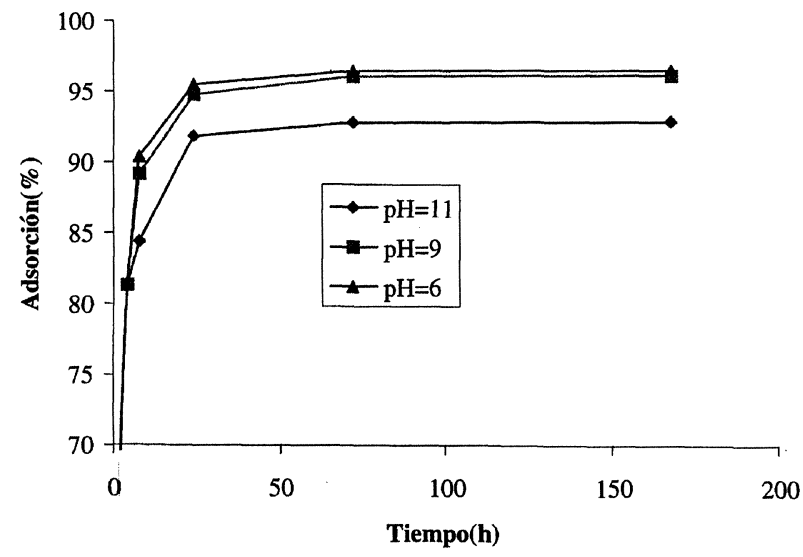

Figura 7. Cinética de adsorción de oro en carbón activado en disoluciones saturadas en oxígeno disuelto, $(0,8 \mathrm{mg} / \mathrm{l})$, en función del $\mathrm{pH}$ de la disolución, y para una concentración inicial de potasio de 0,5 M.

Figure 7. Kinetics of gold adsorption on activated carbon from oxygen saturated $10,8 \mathrm{mg} / \mathrm{l})$ aqueous solutions of various $\mathrm{pH}$ and initial potassium concentration of $0,5 \mathrm{M}$. 


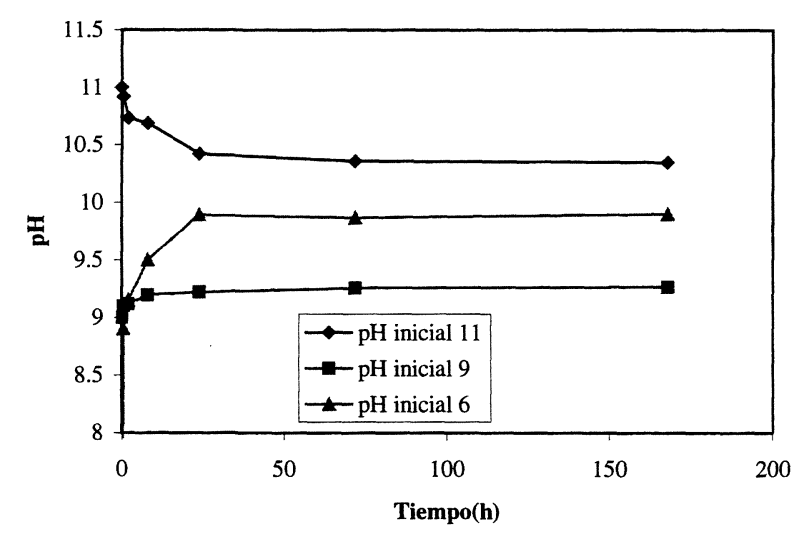

Figura 8. Evolución del pH en la adsorción de oro en carbón activado para una concentración de $0,5 \mathrm{M}$ de potasio, 0,8 $\mathrm{mg} / \mathrm{l}$ de oxígeno disuelto y diferentes $\mathrm{pH}$ iniciales.

Figure 8. Evolution of $\mathrm{pH}$ in gold adsorption on actived. carbon. Initial potassium concentration $0,5 \mathrm{M}$, dissolved oxygen, 0,8 mg/l and differents initials $\mathrm{pH}$.

observa un aumento de la adsorción de oro al disminuir el pH de la disolución. Si se compara la adsorción de oro después de 168 h de contacto, para los diferentes $\mathrm{pH}$ empleados bajo las condiciones de disoluciones saturadas en oxígeno $(8 \mathrm{mg} / \mathrm{l}$ de oxígeno disuelto) y disoluciones desoxigenadas (0,8 mg/l de oxígeno disuelto), se observa en todos los casos que la adsorción de oro aumenta al trabajar con disoluciones saturadas en oxígeno, diferencia que se hace mas evidente para tiempos cortos de adsorción, como se puede observar en la tabla III. Como se observa en las figuras 5 y 7 , la adsorción de oro aumenta al trabajar con $\mathrm{pH}$ bajos, tanto para disoluciones saturadas en oxígeno como desoxigenadas, para una concentración de potasio

Tabla III. Adsorción de oro en función del pH y concentración de oxígeno disuelto

Table III. Gold adsorption at various $\mathrm{pH}$ and dissolved oxygen concentrations

\begin{tabular}{ccrc}
\hline $\begin{array}{c}\text { Oxígeno disuelto } \\
(\mathrm{mg} / \mathrm{l})\end{array}$ & Tiempo (h) & $\mathrm{pH}$ & Adsorción (\%) \\
\hline \multirow{2}{*}{8} & 8 & 11 & 80,73 \\
& & 9 & 93,00 \\
& 168 & 11 & 93,82 \\
& & 9 & 98,81 \\
& & 6 & 98,62 \\
& & 11 & 84,36 \\
& 8 & 9 & 89,09 \\
& & 6 & 90,36 \\
& & 11 & 92,93 \\
& 168 & 9 & 96,16 \\
& & 6 & 96,64 \\
\hline
\end{tabular}

de 0,5 M. Debido a la elevada concentración de potasio presente, el mecanismo de adsorción de oro predominante es el de par iónico, como $\mathrm{M}^{\mathrm{a}+}\left[\mathrm{Au}(\mathrm{CN})_{2}^{-}\right]_{\mathrm{n}}$, donde $\mathrm{M}^{\mathrm{n}+}$ es, en este caso, $\mathrm{K}^{+}$. Cuando se experimentó con disoluciones de pH 6 y 9 , al aumentar la concentración de $\mathrm{H}^{+}$, se favoreció la adsorción del par iónico $\mathrm{HAu}(\mathrm{CN})_{2}$, en conjunto con el par iónico $\mathrm{KAu}(\mathrm{CN})_{2}$, lo cual es consistente con el aumento de $\mathrm{pH}$ de la disolución y la mayor adsorción de oro obtenida bajo esta condición. La capacidad de carga de un carbón activado aumenta con la concentración y tipo de cationes en solución, en el siguiente orden: $\mathrm{Ca}^{2+}>$ $\mathrm{Mg}^{2+}>\mathrm{H}^{+}>\mathrm{Li}^{+}>\mathrm{Na}^{+}>\mathrm{K}^{+}$, lo cual es concordante con lo encontrado en este estudio, para este tipo de carbón activado.

Por otra parte queda de manifiesto que la formación de sitios activos de intercambio de iones a diferentes $\mathrm{pH}$, para disoluciones saturadas en oxígeno con respecto a disoluciones desoxigenadas, es péqueña al emplear concentraciones de potasio de $0,5 \mathrm{M}$, ya que se produjo un aumento del $4 \%$ de adsorción de oro a pH 11, $2 \%$ de aumento a pH 9 y $2 \%$ de aumento a $\mathrm{pH} 6$, lo cual evidencia la importancia del mecanismo del par iónico en disoluciones de elevada fuerza iónica.

\subsection{Efecto del oxígeno disuelto en función de la concentración de oro en la disolución}

En la figura 9 se ha representado la adsorción de oro en función del tiempo, para dos diferentes concentraciones iniciales de oro de 55 y $105 \mathrm{mg} / \mathrm{l}$ respectivamente, con 8 y $0,8 \mathrm{mg} / \mathrm{l}$ de oxígeno

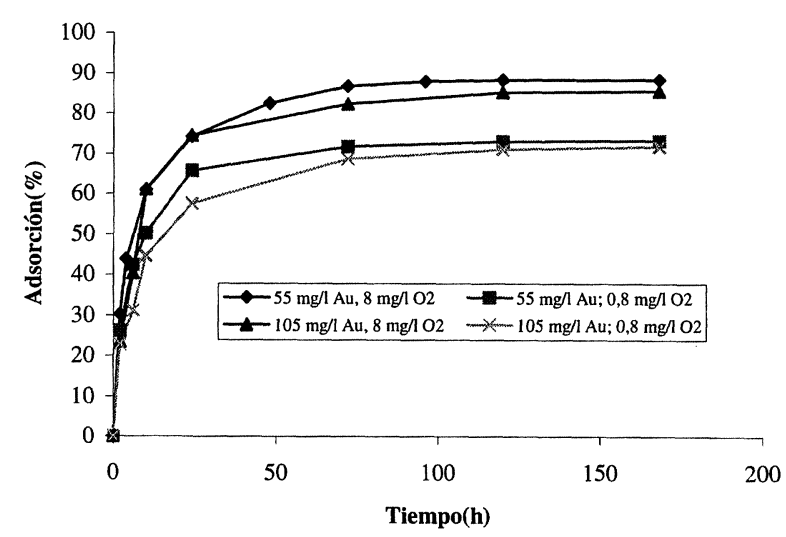

Figura 9. Efecto del oxígeno disuelto en la adsorción de oro en carbón activado para diferentes concentraciones de oro y oxígeno y concentración de potasio de $6\left(10^{-4} \mathrm{M}\right.$.

Figure 9. Effect of the dissolved oxygen in gold adsorption on activated carbon from various gold concentrations and aqueous solutions and $6\left(10^{-4} \mathrm{M}\right.$ potassium initial concentration. 
disuelto, $\mathrm{pH} 11$ y una concentración de potasio de $6 \times 10^{-4} \mathrm{M}$.

Como se discutió en el punto 3.1, para disoluciones de una baja fuerza iónica existe una gran contribución del mecanismo de intercambio de iones en el proceso de adsorción de oro, el cual no cambia al aumentar la concentración inicial del metal de 55 a $105 \mathrm{mg} / \mathrm{l}$. Esto se ve reflejado en el hecho de que, para una concentración inicial de oro de $55 \mathrm{mg} / \mathrm{l}$, el aumento de la extracción máxima al emplear $8 \mathrm{mg} / \mathrm{l}$ de oxígeno disuelto con respecto a la disolución desoxigenada es de $15 \%$, en tanto que, cuando se emplea una concentración inicial de $105 \mathrm{mg} / \mathrm{l}$, el aumento de extracción alcanza a $14 \%$.

Estos resultados reafirman el hecho de que, para una disolución de baja fuerza iónica, independientemente de la concentración inicial de oro en disolución, la adsorción de $\mathrm{Au}(\mathrm{CN})_{2}^{-}$en carbón activado ocurre a través del mecanismo de par iónico, tanto en ausencia como en presencia de oxígeno y además mediante la formación de sitios de intercambio de iones en presencia de oxígeno disuelto.

\section{CONCLUSIONES}

Las conclusiones más importantes de este estudio son las siguientes:

- Cuando se emplean disoluciones saturadas en oxígeno, la adsorción de oro en carbón activado aumenta, siendo este efecto más importante para disoluciones de baja fuerza iónica, y en menor grado, para disoluciones de elevada fuerza iónica.

- Para disoluciones saturadas en oxígeno y disoluciones desoxigenadas con elevada y baja fuerza iónica del medio, el mecanismo mayoritario de adsorción de oro ocurre a través del par iónico del tipo $\mathrm{M}^{\mathrm{a}+}\left[\mathrm{Au}(\mathrm{CN})_{2}^{-}\right]_{\mathrm{n}}$. Esto se ve reflejado fuertemente a un $\mathrm{pH}$ inicial 6 , debido a la presencia de $\mathrm{K}^{+}$y $\mathrm{H}^{+}$en la disolución acuosa, en el aumento del $\mathrm{pH}$ a través del tiempo. En tanto, el mecanismo de intercambio de iones vía activación de sitios superficiales del carbón activado ocurre, fundamentalmente, con disoluciones saturadas en oxígeno disuelto y de baja fuerza iónica, en conjunto con el mecanismo de adsorción de par iónico, lo cual se ve reflejado en la mayor adsorción de oro alcanzada con relación a la condición experimental de elevada fuerza iónica.

- El pH de la disolución acuosa y la concentración inicial de oro no modifica los mecanismos operantes en la adsorción de oro en carbón activado, al emplear disoluciones saturadas en oxígeno y desoxigenadas, con alta y baja fuerza iónica.

\section{REFERENCIAS}

[1] R. Merello y G. Zárate, Revista Minerales 42 (1987) 25-33.

[2] Van Deventer y P. Van Der Merwe, Metall. Trans. B 25B (1992) 829-838.

[3] N. VeGTeR y N. SANDERBERGH, Hydrometallurgy 28 (1992) 205-222.

[4] G. MC Dougall., J. S. Afr. Inst. Min. Metall. 91 (1991) 109-120.

[5] C. Fleming y M. Nicol, J. S. Afr. Inst. Min. Metall. 84 (1984) 85-93.

[6] S. CARello y A. VIDELA, $49^{\circ}$ Congreso Anual, Tecnología Mineral Tratamento da Resíduos de Processos, vol. IVSao Paulo, Brasil, 1994, pp. 401 - 414.

[7] I. IBRADO Y C. FueRTENAU, Hydrometallurgy 30 (1992) 243-256.

[8] M. Adams y C. Fleming, Metall. Trans. B 10B (1978) 165-169.

[9] M. Adams, G. MC Dougall y R. Hancock, Hydrometallurgy 19 (1987) 95-115.

[10] M. Adams, G. Mc Dougall y R. Hancock, Hydrometallurgy 18 (1987) 139-154.

[11] M. AdAMs, Hydrometallurgy 25 (1990) 171-184.

[12] C. KLAUBER Y C. VERNON, Hydrometallurgy 25 (1990) 387-396.

[13] G. Mc Dougall y R. Hancock, Gold Bull. 14 (1981) 138-153.

[14] M. Adams y C. Fleming, Metall. Trans. B 2OB (1989) 315-325.

[15] N. TSUCHIDA y D. MUIR, Metall. Trans. B 17B (1986) 523-528.

[16] N. TsUCHIDA y D. MUIR, Metall. Trans. B 17B (1986) 529-533. 